\title{
Virtual Reality Exposure Therapy (VRET) for Anxiety Due to Fear of COVID-19 Infection: A Case Series
}

This article was published in the following Dove Press journal: Neuropsychiatric Disease and Treatment

\author{
Wanling Zhang ${ }^{1,2, *}$ \\ Dhirendra Paudel (D) ${ }^{2,3, *}$ \\ Rui Shi ${ }^{1}$ \\ Jie Liang' \\ Jingwen Liu' \\ Xiansheng Zeng ${ }^{\prime}$ \\ Yunfei Zhou' \\ Bin Zhang $\mathbb{D}^{2,4}$
}

\begin{abstract}
'Shenzhen Mental Health Center, Shenzhen Kangning Hospital, Shenzhen $5|8| 18$, People's Republic of China; ${ }^{2}$ Center for Brain Science and BrainInspired Intelligence, Guangdong-Hong Kong-Macao Greater Bay Area, Southern Medical University, Guangzhou, 5I05I5, People's Republic of China; ${ }^{3}$ Department of Psychiatry, Dhaulagiri Hospital, Baglung 33300, Gandaki, Nepal; ${ }^{4}$ Department of Psychiatry, Nanfang Hospital, Southern Medical University, Guangzhou 5105I5, People's Republic of China
\end{abstract}

*These authors contributed equally to this work

Correspondence: Bin Zhang Department of Psychiatry, Nanfang Hospital, Southern Medical University, Guangzhou 5105 I5, People's Republic of China Tel +86-20-6278673।

Email zhang73bin@hotmail.com

Yunfei Zhou

Shenzhen Mental Health Center, Shenzhen Kangning Hospital, Shenzhen 518118 ,

People's Republic of China

Tel +86-755-256I5965

Email drzyf2007@।63.com

\begin{abstract}
Virtual reality exposure therapy (VRET) is becoming popular for treating phobia and anxiety disorder. The recent pandemic of COVID-19 not only causes infection per se but also has an impact on mental health. This case series aimed to explore the role of VRET in the intervention of psychiatric illnesses with chief complaints of fear of COVID-19 infection. In vivo exposure therapy for fear of COVID-19 infection is not possible due to the risk of virus infection; in this scenario, the VRET provides an immersive experience and can act as adjunctive therapy for treating phobias and anxiety disorders arising due to novel coronavirus pandemic. Clinical presentation and findings as well as management and procedures of VRET are discussed. Medical record of three patients (two male and one female) at the Shenzhen Mental Health Center (Shenzhen Kangning Hospital), China, was included in the present case series. Patients were assessed with the Hamilton Anxiety Rating Scale and Fear of COVID-19 Scale to measure anxiety and fear, respectively. Throughout VRET sessions, we gradually and systematically exposed the patient to virtual COVID-19 scenarios (for example, touching stained door handle which may have viruses, watching pandemic news, watching frontline health care workers, etc.). In our study, VRET intervention significantly reduced the related symptoms caused by fear of COVID-19 infection. Furthermore, virtual reality can provide relevant theoretical and practical support for exploring the remote psychological counseling of patients in isolation wards.
\end{abstract}

Keywords: VRET, COVID-19, phobia, telemedicine, telehealth, exposure therapy

\section{Introduction}

The novel coronavirus (COVID-19) has spread globally. As of August 16, 2020, there were 21,294,845 confirmed cases and 761,779 deaths worldwide. ${ }^{1}$ The pandemic has brought not only viral infection and associated death but also psychological distress and mental disorders. ${ }^{2}$ The emergence of the COVID-19 pandemic has exacerbated fear around the world. ${ }^{3}$ People fear of being infected with COVID19 , being a source of infection, discrimination, and stigmatization, and contracting COVID-19 and transmitting it to elderly family members. ${ }^{4}$ Due to high levels of fear, individuals may show irrational behaviors while dealing with COVID-19. ${ }^{3}$ Recently, the inevitable coronavirus outbreak has entered into a spectrum of phobias termed coronaphobia where overt apprehension and overreaction towards COVID-19 are likely to have unpleasant and hyperarousal symptoms triggered by information or cognitions about COVID-19 infection. ${ }^{5}$ 
However, the current global treatment of COVID-19 mainly focuses on infection and its management. The psychosocial aspects of this pandemic are serious and affect different social strata. ${ }^{6}$ As countries around the world must work hard to reduce the spread of COVID19 , they should also work hard to eliminate personal fears to achieve the overall goal of building a COVID-19-free society. ${ }^{3}$ This unique situation requires a dynamic approach of treating not only infected patients but also patients with fear of infection.

During the pandemic, virtual reality (VR) is rapidly changing the medical training, patient treatments, and awareness about COVID-19. ${ }^{7}$ The pandemic has transformed the medical care system with virtual telemedicine and software platforms to deliver medical services to mitigate the effects of COVID-19. ${ }^{7,8}$ There is a rapid adaptation of digital tools and technologies in healthcare services. ${ }^{9}$ For fear of COVID-19 infection, the pandemic poses a difficult task for in vivo exposure wherein virtual reality exposure therapy (VRET) can be a safe effective alternative with equal efficacy.

In the past, it has been shown that exposure in vivo has a better outcome than imaginal exposure for anxiety disorder especially phobias. ${ }^{10}$ When exposure is successful more new and neutral memory structures are created which overturn the anxiety triggers. ${ }^{10}$ It has been shown that virtual reality exposure therapy (VRET) was effective in exposure therapy, ${ }^{11}$ which can be used to elicit fear and inducing anxiety. The experience which discords the anxious belief leads to a decrease in anxiety and thus leads to habituation. ${ }^{10}$ VRET has gained its popularity in treating phobia and anxiety as an alternative to exposure in vivo. ${ }^{12}$

The advantages of VRET include an immersive experience, economical, replicable, and more realistic than imaginal exposure with flexible operability and availability. ${ }^{10,13,14}$ In the COVID-19 outbreak, it is highly safe, easily acceptable, and avoids the risk of infection in the exposure process. The current study aimed to explore the usefulness of VRET to treat irrational fear of COVID-19.

\section{Materials and Methods}

\section{Patients}

Three patients treated at Shenzhen Mental Health Center (Shenzhen Kangning Hospital) with chief complaints of fear of COVID-19 infection were included in this case series.
All three patients went through extensive physical examination as well as routine laboratory examinations including electroencephalogram, electrocardiogram, and echocardiogram before exposure therapy. On further psychiatric evaluation, patients met the diagnostic criteria for generalized anxiety disorder according to the international classification of diseases, tenth edition. ${ }^{15}$ All the patients were treated as usual with antidepressants and benzodiazepines for more than two weeks before the VRET intervention.

\section{Assessment Method}

The quantitative assessment was carried out by selfreporting questionnaires applied at admission, before starting VRET intervention and after the final VRET session. 14-item Hamilton Anxiety Scale (HAMA) ${ }^{16}$ was used to measure anxiety symptoms in which each item is scored on a scale of 0 to 4 with total score ranges from 0 to 56 . 7-item Fear of COVID-19 scale (FCV-19S) ${ }^{3}$ was used to measure phobic symptoms with a five-item Likert type scale in which each item is scored on a scale of 1 to 5 and the total score ranges from 7 to 35 . The higher the score, the greater the severity of anxiety and fear of COVID-19 respectively. Repeated measure ANOVA was carried out to compare the scores of HAMA and FCV-19s before the patients began VRET, and after completing the final VRET session. The qualitative assessment was done using a clinical interview and observing behavior.

\section{VRET}

\section{Equipment}

An Extended Realistic Exposure Therapy System (XRET 1.0) workstation manufactured by Beijing Xinxi Health Technology Co., Ltd with Intel Core i7-9700 Hexa core processor CPU, 16 GB DDR4 RAM, 2TB storage HDD, 256 GB NVME system SSD, 8 GB graphics card RTX2070, and HTC VIVE Pro Eye Professional headgear.

\section{Virtual Coronavirus Situation}

After the application of the headgear, the patient can see and have an immersive experience for various scenarios of the COVID-19 pandemic (stains on door handle which may have viruses, watching pandemic news, watching frontline health care workers, health care worker measuring body temperature on others, pick up the thermal gun to measure temperature on the patient, coughing pedestrians on the street, coughing passenger on the subway, and doctors doing consultation in isolation ward). The 
psychiatrist and nurse can also see simultaneously on a screen what the patient is experiencing. A list of scenarios and exposure factors used in VRET is shown in Table 1.

\section{Intervention}

The following procedures were implemented for applying VRET intervention:

(1) Preparation: The patient was prepared for VRET by a psychiatrist and a nurse with psychoeducation regarding exposure principle and protocol. Furthermore, motivational interviews, queries regarding exposure were answered, and preheating was done. The assessment of the physical condition of the patient was done to ensure no health risk during exposure therapy.

(2) Graded exposure stimulus: A psychiatrist and nurse develop a list of graded exposure levels after interviewing the patient. Preliminary exposure scenario screening was conducted and the degree of anxiety caused by exposure stimuli was graded. Patient education was done on how to use the Subjective Units of Distress Scale (SUDS). ${ }^{17}$

(3) The course of treatment: Patients were evaluated during treatment with SUDS. The scale ranges from 1 to 10. The exposure was stopped when the patient's SUDS score in all modalities was below 4. If there was no relaxation after 40 minutes, exposure was stopped. The relaxing scene was kept as a backup. If the patient feels overstressed, the patient was helped with the relaxing scene.

(4) First exposure: The patient was first introduced to exposure stimuli of the low-grade stimulus. The patient was exposed and continued to receive stimulus until there was no avoidance or ritualized behavior in response to anxiety. The patient rated distress during VRET using SUDS and exposure stimulus was stopped after desensitization occurs.

(5) Repeated exposure: Repeated exposure tasks with the same stimulus was given until self-rated SUDS halved. Then the new exposure stimulus entry for exposure was introduced. Exposure therapy was usually conducted gradually from low-grade exposure stimuli to high-anxiety stimuli until the patient's peak anxiety SUDS score in all scenarios was below 4. At the end of therapy, the patient would stop showing the avoidance behavior.

\section{Case Presentations Case I}

A 41-year-old male freelancer by work was admitted to the hospital on June 10, 2020, with complaints of prominent tension, worry, palpitation and restlessness for more than 4 months and aggravated for 1 month. The patient learned of the COVID-19 case in Wuhan on his way home in late January 2020. The patient had excessive and uncontrollable worry about getting a COVID-19 infection. The patient had reassurance-seeking behavior reflected by multiple visits to hospitals to rule out COVID-19 infection. However, patients continue to have an excessive worry of COVID-19 infection and started having symptoms such as restlessness, palpitation, chest tightness, muscle tensions, aches and pains over the whole body, and poor sleep. The patient was unable to work efficiently. The patient was not under medication before admission. With pharmacological treatments sleep improved significantly but the improvements in anxiety symptoms were not

Table I Virtual Coronavirus Situation

\begin{tabular}{|c|c|c|}
\hline Coronavirus Situation & The Main Factor & Exposure Factor \\
\hline Public health room & Door handle (touch) & Stains (may have viruses) \\
\hline \multirow[t]{4}{*}{ Hospital hall } & The waiting area & Watch the news (epidemic) \\
\hline & & Keep an eye on the health care workers \\
\hline & & Health care worker measures body temperature \\
\hline & Test area & Pick up the thermal gun to measure temperature \\
\hline Urban & Street area & Coughing pedestrians \\
\hline Subway station & The platform area & Coughing passenger \\
\hline Hospital ICU & Bed area & Doctors doing consultation \\
\hline Beach* & Music & Music I (Lounge) \\
\hline
\end{tabular}

Note: *Beach area is kept as backup for relaxation therapy. 
significant. The thought of COVID-19 brought nervousness, chest tightness, and restlessness. VRET was started on July 1. After six sessions of virtual exposure therapy, symptoms were reduced markedly. The fear of COVID-19 was significantly reduced. Furthermore, palpitation, chest tightness, restlessness, and other symptoms disappeared. The patient was discharged from the hospital on July 8, 2020. After leaving the hospital, the patient quickly resumed the daily social and occupational activities. During the follow-up, the symptoms of anxiety had disappeared and the patient was able to work and socialize normally.

\section{Case 2}

A 41-year-old male working in information and technology, admitted to the hospital on 2 July 2020 with chief complaints of nervousness and worrying thoughts for 5 months, easy irritability for 3 months which aggravated for more than 1 month. The patient caught a cold during the COVID-19 outbreak and was feverish with a body temperature of $37.1^{\circ} \mathrm{C}$. The patient feared that he contracted COVID-19, but the PCR and lung CT investigations were normal. The patient was still not assured and had symptoms of palpitation, chest tightness, shortness of breath, tremor, bodily discomforts, restlessness, lack of concentration, weight loss of more than 10 pounds, and poor sleep. Social functioning was markedly impaired. On 18 June, the patient was treated with antidepressants and benzodiazepines in another hospital. Patients feared that the medication would stop working itself. He was kept on an antidepressant and benzodiazepine treatment after admission. The symptoms of anxiety improved while the patient was admitted to the hospital. Patients considered the hospital environment to be clean and safe and he was afraid to leave the hospital. The patient feared contracting coronavirus from the passers-by and the fear was not improved with medications. VR exposure therapy was started on July 4. There was a rapid improvement after two exposures. The patient was discharged on July 16, 2020. After being discharged from the hospital, the patient quickly returned to normal social life and work. During followup, the self-reported anxiety symptoms had largely disappeared. The patient worried about novel coronavirus but there was no impairment in daily activities and work.

\section{Case 3}

A 55-year-old female, retired finance analyst admitted to the hospital on 28 June 2020 presented with increased nervousness, worry, palpitation, chest tightness, and poor sleep for more than 1 year, aggravated for more than 3 months. The illness was mild one year ago and no systematic treatment was given. The novel Coronavirus pandemic aggravated the situation. She had a son who lives and works in New York, USA. The disease worsened significantly and she often called her son in the early hours of the morning to confirm the safety of her son. She worried that she and her son would contract "novel Coronavirus". She had symptoms of easy nervousness, excessive worry, worry about all kinds of small things, restlessness, accompanied by palpitation, chest tightness, breathing difficulties, trembling, low mood, and a decline in interest. The social function was seriously impaired that she seldom got out of her house. She asked her friends to bring her food but not allowing her friends to enter the house. Antidepressant and benzodiazepine therapy were started on June 16. There was an initial improvement in sleep but was not sustained. After admission, patients continued to follow the original medication regimen, and there was a reduction in chest discomfort and palpitation. Yet fear of novel coronavirus remained and the patient kept a distance of more than $1.5 \mathrm{~m}$ from the other patient in the ward. The patient was hypervigilant for use of paper towels when opening the door and pressing the elevator. She had an FCV-19S score of 27. VRET was started on July 2, and the patients had five sessions of VRET. After the final session of VRET, she still had a score of 20 on FCV-19S. But there was a remarkable return to social functioning and a willingness to interact with people and go out. She was able to control her avoidance behavior and was discharged from the hospital on July 22, 2020.

\section{Results}

Table 2 shows the scores of quantitative clinical measurement instruments used during the study. The assessment of anxiety symptoms by HAMA shows a significant decrease $(\mathrm{F}=31.681, \mathrm{P}=0.030)$ in the mean score between the preVRET intervention (Mean $(\mathrm{M})=23.33$, standard deviation $(\mathrm{SD})=7.02)$ and after the final VRET intervention $(\mathrm{M}=7.67, \mathrm{SD}=2.89)$. There was a reduction in the mean score of FCV-19S which measured phobic symptoms before VRET intervention $(\mathrm{M}=26.67, \mathrm{SD}=2.52)$ and after the final VRET intervention $(\mathrm{M}=13.33, \mathrm{SD}=5.86)$ but the VRET effect was not statistically significant $(\mathrm{F}=12.598$, $\mathrm{p}=0.071$ ). However, qualitatively there was a reduction in avoidance behavior and improvement in social 
Table 2 Scores of Anxiety and Phobic Symptoms

\begin{tabular}{|l|l|l|l|l|l|l|l|}
\hline Cases & $\begin{array}{l}\text { Age } \\
\text { (Years) }\end{array}$ & Gender & HAMA & \multicolumn{2}{l|}{ FCV-19S } \\
\hline & & & $\begin{array}{l}\text { At the Time of } \\
\text { Admission }\end{array}$ & $\begin{array}{l}\text { Before } \\
\text { Exposure }\end{array}$ & $\begin{array}{l}\text { After } \\
\text { Exposure }\end{array}$ & $\begin{array}{l}\text { Before } \\
\text { Exposure }\end{array}$ & $\begin{array}{l}\text { After } \\
\text { Exposure }\end{array}$ \\
\hline Case I & 41 & M & 38 & 24 & 6 & 29 & 9 \\
Case 2 & 41 & M & 30 & 16 & 6 & 24 & 11 \\
Case 3 & 55 & F & 40 & 30 & 11 & 27 & 20 \\
\hline
\end{tabular}

Notes: M, Male; F, Female; HAMA, 14-item Hamilton Anxiety Scale (total score range: 0-56); FCV-I9S, 7-item Fear of COVID-19 Scale (total score range: 7-35).

functioning. Clinically there was a reduction in anxiety symptoms and patients were able to calibrate the fear of COVID-19.

\section{Discussion}

During the COVID-19 pandemic, there is a broad range of psychological responses with patients expressing symptoms of stress, anxiety, insomnia, fear, and depression. ${ }^{18}$ Some people feel preoccupied with the notion of lockdown and overwhelm themselves while others report excessive concerns of infection to them and their families, moreover, still some fear of infection and death from the disease. ${ }^{2,18,19}$ While the situation of the COVID-19 outbreak may be novel, the subject of isolation and fear is not.

In these three patients, we observed that the physical discomfort of the patients improved rapidly after pharmacological treatment, but the excessive fear of COVID-19 had not been alleviated. The social and occupational function was still affected. And the duration of the COVID-19 pandemic is unknown and this uncertainty can exacerbate the phobic anxiety symptoms. Exposure therapy is a component of behavioral therapy in which patients are exposed to something that they are afraid of most. ${ }^{10}$ People who are anxious about coronavirus do not need to be exposed to the virus. Thanks to virtual reality technology, it can be simulated and patients can be exposed to various situations to eliminate emotional anxiety in a diverse group of anxiety disorders. ${ }^{11}$

The use of VRET has brought technologically driven exposure therapy which can be utilized to remove phobias related to COVID-19. In our study after VRET intervention patients were able to calibrate their fear and there was also a significant improvement in social and occupational functioning. Furthermore, VRET can be used to reduce the feeling of isolation that persists during the pandemic phase. When used in conjunction with medication it can remove the lingering psychological components of anxiety.

VRET has achieved success in reducing anxiety symptoms, which provides evidence for the effectiveness of VRET as a new medium of exposure therapy in recent research studies. ${ }^{11}$ In the virtual coronavirus world, patients experience various social and medical conditions, retrieve and modify their stored memories. VRET is believed to work by modifying the patient's memory. ${ }^{10}$ After treatment, patients can recall in more detail what happened during the pandemic without the same level of fear. This will no longer cause anxiety symptoms. The advantage of overexposure therapy is that it allows the patient to experience the pandemic in a controlled way, thereby forming a habit. The patients seemed to be immersed in a virtual environment. It should be noted that VRET has helped improve the cognitive components in our cases probably by lessening negative cognitions. ${ }^{12}$

VRET was able to remove the fears of infection and improve social interaction. In the treatment of phobias, virtual reality technology can provide an external environment for patients who cannot tell their own experiences, where they can encounter and control their trauma. ${ }^{20}$ The multiple sensory cues provided by virtual reality may also provide greater possibilities to involve patients and create a sense of presence, thereby facilitating the processing of experiences related to triggering anxiety. ${ }^{13}$ The VRET environment can be manipulated under the constraints of the daily world, thereby creating new possibilities for therapeutic behavior. ${ }^{13}$

Pandemic poses challenges of providing medical services in isolation wards due to shortages of protective tools which increases the risk of infection to patients and treating physicians. ${ }^{9}$ The health care professionals working in isolation wards are suffering from anxiety, stress, and fear. ${ }^{9,18}$ Due to a lack of proper training for working in isolation wards mental health care providers are at risk of 
infection. The VR treatment modalities can help in these situations if there are necessary tools available. The psychological assistance can be provided remotely with virtual reality and software platforms. ${ }^{7,9,18}$ The VR technology can help provide remote guidance and psychological assistance as well as can mitigate the risk of infection to the patient and mental healthcare providers.

\section{Conclusion}

In conclusion, the results of this case series indicate that VRET is safe and effective in enhancing the treatment of fear of COVID-19 and associated anxiety disorder. There is a significant reduction in avoidance behavior after VRET intervention. With an increase in the affordability of VR tools, researchers can evaluate its role in routine therapy. However, a trained therapist and well-programmed stimuli are necessary to guide the treatment procedures.

\section{Limitation}

There are lots of limitations of case studies in research domains. Although case studies are useful in illustrating imaginative advances, the efficacy of which needs to be further explored. Cybersickness, the adverse effect of VRET, in some patients may limit the immersive exposure and usefulness of VRET. Few mental health providers are trained in VRET. Furthermore, well coordination is required between virtual reality technologists and medical professionals to create appropriate exposure scenarios. Given the potential of VRET and the need for new technologies to treat the psychological consequences of the COVID-19 pandemic, it is necessary to further study the potential value of VRET exposure therapy to treat the fear of the COVID-19 outbreak.

\section{Ethical Consideration}

Written informed consent was taken from the patients to publish the case reports. The study was approved by the Institutional Review Board of Kangning Hospital.

\section{Funding}

This research was funded by Shenzhen Key Medical Discipline Construction Fund (grant number. SZXK041) and Shenzhen Fund for Guangdong Provincial High-level Clinical Key Specialties (grant number. SZGSP013).

\section{Disclosure}

The authors report no conflicts of interest in this work.

\section{References}

1. World Health Organization. Coronavirus Disease (COVID-2019): Situation Report-209. 2020.

2. Cao W, Fang Z, Hou G, et al. The psychological impact of the COVID-19 epidemic on college students in China. Psychiatry Res. 2020;287:112934. doi:10.1016/j.psychres.2020.112934

3. Ahorsu DK, Lin CY, Imani V, Saffari M, Griffiths MD, Pakpour AH. The fear of COVID-19 scale: development and initial validation. Int $J$ Ment Health Addict. 2020;1-9.

4. Lin C-Y. Social reaction toward the 2019 novel coronavirus (COVID-19). J Health Soc Behav. 2020;3(1):1. doi:10.4103/SHB. SHB_11_20

5. Lee SA, Jobe MC, Mathis AA, Gibbons JA. Incremental validity of coronaphobia: coronavirus anxiety explains depression, generalized anxiety, and death anxiety. J Anxiety Disord. 2020;74:102268. doi:10.1016/j.janxdis.2020.102268

6. Dubey S, Biswas P, Ghosh R, et al. Psychosocial impact of COVID-19. Diabetes Metab Syndr. 2020;14(5):779-788. doi:10.1016/j.dsx.2020.05.035

7. Singh RP, Javaid M, Kataria R, Tyagi M, Haleem A, Suman R. Significant applications of virtual reality for COVID-19 pandemic. Diabetes Metab Syndr. 2020;14(4):661-664. doi:10.1016/j. dsx.2020.05.011

8. Bokolo AJ. Exploring the adoption of telemedicine and virtual software for care of outpatients during and after COVID-19 pandemic. Ir J Med Sci. 2020. doi:10.1007/s11845-020-02299-z

9. Bokolo Anthony J. Use of telemedicine and virtual care for remote treatment in response to covid-19 pandemic. J Med Syst. 2020;44 (7):132. doi:10.1007/s10916-020-01596-5

10. Krijn M, Emmelkamp PMG, Olafsson RP, Biemond R. Virtual reality exposure therapy of anxiety disorders: a review. Clin Psychol Rev. 2004;24(3):259-281.

11. Carl E, Stein AT, Levihn-Coon A, et al. Virtual reality exposure therapy for anxiety and related disorders: a meta-analysis of randomized controlled trials. $J$ Anxiety Disord. 2019;61:27-36. doi:10.1016/j.janxdis.2018.08.003

12. Meyerbroker K, Emmelkamp PM. Virtual reality exposure therapy in anxiety disorders: a systematic review of process-and-outcome studies. Depress Anxiety. 2010;27(10):933-944. doi:10.1002/ da. 20734

13. Difede J, Hoffman HG. Virtual reality exposure therapy for World Trade Center post-traumatic stress disorder: a case report. Cyberpsychol Behav. 2002;5(6):529-535. doi:10.1089/ 109493102321018169

14. Opris D, Pintea S, Garcia-Palacios A, Botella C, Szamoskozi S, David D. Virtual reality exposure therapy in anxiety disorders: a quantitative meta-analysis. Depress Anxiety. 2012;29(2):85-93. doi:10.1002/da.20910

15. World Health Organization. The ICD-10 Classification of Mental and Behavioural Disorders: Clinical Descriptions and Diagnostic Guidelines. Geneva: World Health Organization; 1992.

16. Hamilton M. The assessment of anxiety states by rating. Br J Med Psychol. 1959;32(1):50-55. doi:10.1111/j.2044-8341.1959.tb00467.x

17. McCabe RE. Subjective units of distress scale. Phobias. 2015;18:361.

18. Kang L, Li Y, Hu S, et al. The mental health of medical workers in Wuhan, China dealing with the 2019 novel coronavirus. Lancet Psychiatry. 2020;7(3):e14. doi:10.1016/S2215-0366(20)30047-X

19. Bao Y, Sun Y, Meng S, Shi J, Lu L. 2019-nCoV epidemic: address mental health care to empower society. Lancet. 2020;395(10224): e37-e38. doi:10.1016/S0140-6736(20)30309-3

20. Klinger E, Légeron P, Roy S, Chemin I, Lauer F, Nugues P. Virtual reality exposure in the treatment of social phobia. Stud Health Technol Inform. 2004;99:91-119. 


\section{Publish your work in this journal}

Neuropsychiatric Disease and Treatment is an international, peerreviewed journal of clinical therapeutics and pharmacology focusing on concise rapid reporting of clinical or pre-clinical studies on a range of neuropsychiatric and neurological disorders. This journal is indexed on PubMed Central, the 'PsycINFO' database and CAS, and is the official journal of The International Neuropsychiatric Association (INA). The manuscript management system is completely online and includes a very quick and fair peer-review system, which is all easy to use. Visit http://www.dovepress.com/testimonials.php to read real quotes from published authors.

Submit your manuscript here: https://www.dovepress.com/neuropsychiatric-disease-and-treatment-journal 